\title{
A privação do sono e suas implicações na saúde humana: uma revisão sistemática da literatura
}

\author{
Sleep deprivation and its implications in human health: a systematic literature review \\ Privación del sueño y sus implicaciones en la salud humana: una revisión sistemática de \\ la literatura
}

Ruan Teixeira Lessa ${ }^{1 *}$, Lucas Augusto Niess Soares Fonseca ${ }^{1}$, Vitória Leite Silva ${ }^{1}$, Francielle Bianca Moreira de Mesquita ${ }^{1}$, Ana Júlia Rodrigues da Costa1, Danilo José Martins de Souza1, Marcelo Ribeiro Cesar ${ }^{1}$, Tayná Beato Ferreira ${ }^{1}$, Luiz Henrique Salamoni Abad ${ }^{1,2}$, Nathália Barbosa do Espírito Santo Mendes ${ }^{1,3}$.

\section{RESUMO}

Objetivo: Analisar, através de uma revisão sistemática da literatura, o impacto do déficit de sono sobre a saúde ao longo de todas as fases da vida e associá-lo ao aparecimento de diversas doenças. Métodos: Foi realizada revisão literária nas bases de dados científicos da área médica, como Latindex, Medline e Lilacs, com os descritores "Transtornos do SonoVigília", "Privação do Sono", "Fases do Sono", "Sono de Ondas Lentas", "Sono REM", e seus correspondentes em inglês e espanhol. Ao final da análise, 29 textos preencheram os parâmetros de seleção estabelecidos. Resultados: A má qualidade do sono em grávidas está associada a maior risco de partos prematuros. Ademais, decréscimos no tempo de sono em crianças tendem a acarretar telômeros mais curtos, que aumentam mortalidade e morbidade na idade adulta. $\mathrm{Na}$ adolescência, o sono insuficiente influencia os padrões alimentares e de atividade física, repercutindo na sua saúde e no seu desenvolvimento. No adulto, desencadeiam-se doenças cardiovasculares, obesidade, diminuição da fertilidade, transtornos psiquiátricos e alterações epigenéticas indesejadas. Já o sono perturbado no idoso pode levar a quedas noturnas intensificadas. Considerações finais: A privação do sono está comprovadamente associada ao desenvolvimento sistêmico de diversas doenças em todas as fases da vida.

Palavras-chave: Transtornos do sono-vigília, Privação do sono, Fases do sono, Sono de ondas lentas, Sono REM.

\section{ABSTRACT}

Objective: Analyze, through a systematic literature review, the impact of sleep deficit on health throughout all stages of life and associate it with the appearance of several diseases. Methods: A literary review was carried out in the scientific databases of the medical area, such as Latindex, Medline and Lilacs, with the descriptors "Sleep-Wake Disorders", "Sleep Deprivation", "Sleep Stages", "Sleep Slow-Wave", "Sleep REM", and their correspondents in Portuguese and Spanish. At the end of the analysis, 29 texts met the established selection parameters. Results: Poor sleep quality in pregnant women is associated with a higher risk of premature births. In addition, decreases in sleep time in children tend to lead to shorter telomeres, which increase mortality and morbidity in adulthood. In adolescence, insufficient sleep influences eating and physical activity patterns, affecting their health and development. Cardiovascular diseases, obesity, decreased fertility, psychiatric disorders and unwanted epigenetic changes are triggered in adults. Disturbed sleep in the elderly can lead to intensified night falls. Final considerations: Sleep deprivation is proven to be associated with the development of systemic diseases at all stages of life.

Key words: Sleep-wake disorders, Sleep deprivation, Sleep stages, Sleep slow-wave, Sleep REM.

\section{RESUMEN}

Objetivo: Analizar, a través de una revisión sistemática de la literatura, el impacto del déficit de sueño acerca de la salud en todas las etapas de la vida y asociarlo a la aparición de varias enfermedades. Métodos: Se realizó una revisión literaria en bases de datos científicas en el campo de la medicina, como Latindex, Medline y Lilacs, con los descriptores "Trastornos del Sueño-Vigilia", "Privación del Sueño", "Fases del Sueño", "Sueño de Onda Lenta", "Sueño REM", y sus correspondientes en inglés y portugués. Al final del análisis, 29 textos cumplieron los parámetros de selección establecidos. Resultados: La mala calidad del sueño en mujeres embarazadas se asocia a un mayor riesgo de parto prematuro. Además, la disminución del tiempo de sueño en los niños tiende a conducir hasta telómeros más cortos, lo que aumenta la mortalidad y la morbilidad en la edad adulta. En la adolescencia, la falta de sueño influye en los patrones de alimentación y actividad física, lo que afecta su salud y desarrollo. En adultos, se desencadenan enfermedades cardiovasculares, obesidad, disminución de la fertilidad, trastornos psiquiátricos y cambios epigenéticos no deseados. La

${ }^{1}$ Centro Universitário Presidente Antônio Carlos (UNIPAC), Juiz de Fora - MG. *E-mail: ruan.t.l@hotmail.com ${ }^{2}$ Hospital Monte Sinai de Juiz de Fora, Juiz de Fora - MG.

3 Universidade Federal de Juiz de Fora (UFJF), Juiz de Fora - MG. 
turbidez de los sueños en los ancianos puede conducir a caídas nocturnas más frecuentes. Consideraciones finales: Se ha demostrado que la falta de sueño está asociada con el desarrollo sistémico de diversas enfermedades en todas las etapas de la vida.

Palabras-clave: Trastornos del sueño-vigilia, Privación del sueño, Fases del sueño, Sueño de onda lenta, Sueño REM.

\section{INTRODUÇÃO}

O sono é um processo fisiológico essencial que realiza a manutenção de vários mecanismos inerentes à homeostase humana (TONONI G e CIRELLI C, 2006). Por esse ângulo, sua privação acarreta alterações significativas no funcionamento físico, ocupacional, cognitivo e social do indivíduo, além de comprometer substancialmente a qualidade de vida (JANSEN JM, et al., 2007).

Além disso, os sonhos também parecem ser marcadores essenciais para um sono de qualidade. Sua importância está relacionada ao desenvolvimento da criatividade e da resolução de problemas. Durante os sonhos, as sinapses neuronais buscam por conexões distantes e infrequentes, fazendo com que informações antes nunca associadas, passem a fazer sentido juntas (WALKER M, 2018).

Dentre os elementos que induzem ao sono estão a melatonina e a adenosina. A primeira é secretada pela glândula pineal e funciona como um sinalizador da presença de luz no ambiente, ou seja, seus níveis no Sistema Nervoso Central (SNC) são muito baixos por volta do meio-dia, mas muito altos por volta da meianoite. Em contrapartida, a adenosina, um neuromodulador que reduz a necessidade de energia pelas células nervosas, pode ser considerada como uma espécie de "pressão" do sono. Essa substância é removida quase que por completo do SNC durante os períodos de sono, mas sua concentração aumenta gradualmente à medida em que permanecemos em vigília (JANSEN JM, et al., 2007).

Outro indutor importante do sono é o ciclo circadiano. Seu significado deriva do latim "circa diem", que significa "cerca de um dia". Esse ciclo é gerenciado pelo núcleo supraquiasmático, um pequeno e difuso conglomerado de células neuronais no hipotálamo anterior, e funciona como relógio biológico endógeno de cerca de 24 horas que regula diversos processos fisiológicos, dentre eles o sono, organizando-os cronologicamente durante o dia (WALKER M, 2018).

Estruturalmente, sabe-se que o sono possui duas subdivisões principais: REM (rapid eye movement) e NREM (non-rapid eye movement). Além disso, a duração de cada uma dessas fases é cíclica e varia de acordo com a evolução das horas, sendo que normalmente se tem mais NREM nas primeiras horas de sono e mais REM nas últimas horas da madrugada (WALKER M, 2018).

Sendo assim, cada uma dessas subdivisões do sono tem funções distintas para o organismo. O sono NREM promove a restauração do Sistema Nervoso Central (SNC) todas as noites, resfria o cérebro e o corpo como um todo; e revigora o sistema imunitário, sendo que a privação dessa fase implica em efeitos imunossupressores no curto e longo prazo. Já o sono REM promove adaptação a eventos emocionais através dos sonhos, consolida a memória e estimula a criatividade, uma vez que a privação dessa fase do sono resulta em desvios na consolidação de lembranças e na transformação de memória de curto em longo prazo (BRYANT PA, 2004).

Portanto, este artigo visa esclarecer, através de revisão sistemática da literatura, as consequências fisiológicas e patológicas da privação do sono durante as diferentes fases da vida, desde o período fetal até a velhice.

\section{MÉTODOS}

O desenvolvimento desta revisão sistemática foi embasado a partir de um levantamento de dados da literatura, publicados no período compreendido entre 2002 a 2019. As bases de dados científicos utilizados foram Medline, Lilacs, Science Citation Index, SciELO, PUBMED e BVS-Bireme. Nas buscas, os seguintes descritores, em língua portuguesa, inglesa e espanhola, foram considerados: "sono na gestante", "sono no feto", "sono na infância", "sono na adolescência", "sono no adulto", "sono no idoso", "privação do sono", 'neurofisiologia do sono", "sono e doenças". Recorreu-se aos operadores lógicos "AND", "OR", "AND NOT" para combinação dos descritores e termos utilizados para rastreamento das publicações. 
Através desse método de busca e seguindo as recomendações do PRISMA (Principais Itens para Relatar Revisões sistemáticas e Meta-análises), foram identificadas, a princípio, 322 publicações potencialmente elegíveis para integrar esta revisão. Em seguida, distinguiram-se os artigos que atenderam aos seguintes critérios de inclusão: (a) bibliografias eletrônicas compatíveis com os descritores enumerados acima; (b) cronologia a partir do ano 2000; (c) textos completos e resumos do tipo tese, artigos originais, de revisão sistemática, livros, instruções normativas e portarias de órgãos científicos oficiais, obtidos nas plataformas indexadoras e bases de dados científicos da área médica supracitados; (d) consideração apenas pelas decorrências da privação do sono. Optou-se por não incluir artigos revisionais narrativos, visto que esses não contemplam a criticidade analítica estimada pelo estudo e também os fatores estatisticamente raros interferentes na fisiologia do sono e suas consequências positivas não foram abrangidos.

Após a primeira análise, com avaliação dos títulos, 298 artigos foram considerados selecionáveis para a segunda fase desta revisão, que consistiu da leitura dos resumos. Após a realização do parecer dos resumos, os estudos que pareciam integrar os critérios de inclusão foram lidos na íntegra. Ao final, 29 referências atenderam a todos os critérios de inclusão e exclusão.

$\mathrm{Na}$ avaliação dos artigos, foram observados os seguintes aspectos: amostra (dimensionamento adequado, envolvendo seleção conveniente de participantes, de acordo com a faixa etária e fase da vida analisada em cada pesquisa); instrumentos (critérios de avaliação); relevância estatística dos estudos; aspectos sistêmicos e patológicos que envolvem a privação do sono e as implicações para a saúde humana; periódico no qual 0 artigo foi publicado (classificados conforme 0 critério Qualis proposto pela CAPES), conforme a Figura 1.

Figura 1 - Identificação e seleção dos artigos sobre a privação do sono na saúde humana para a revisão sistemática, 2002 a 2019.

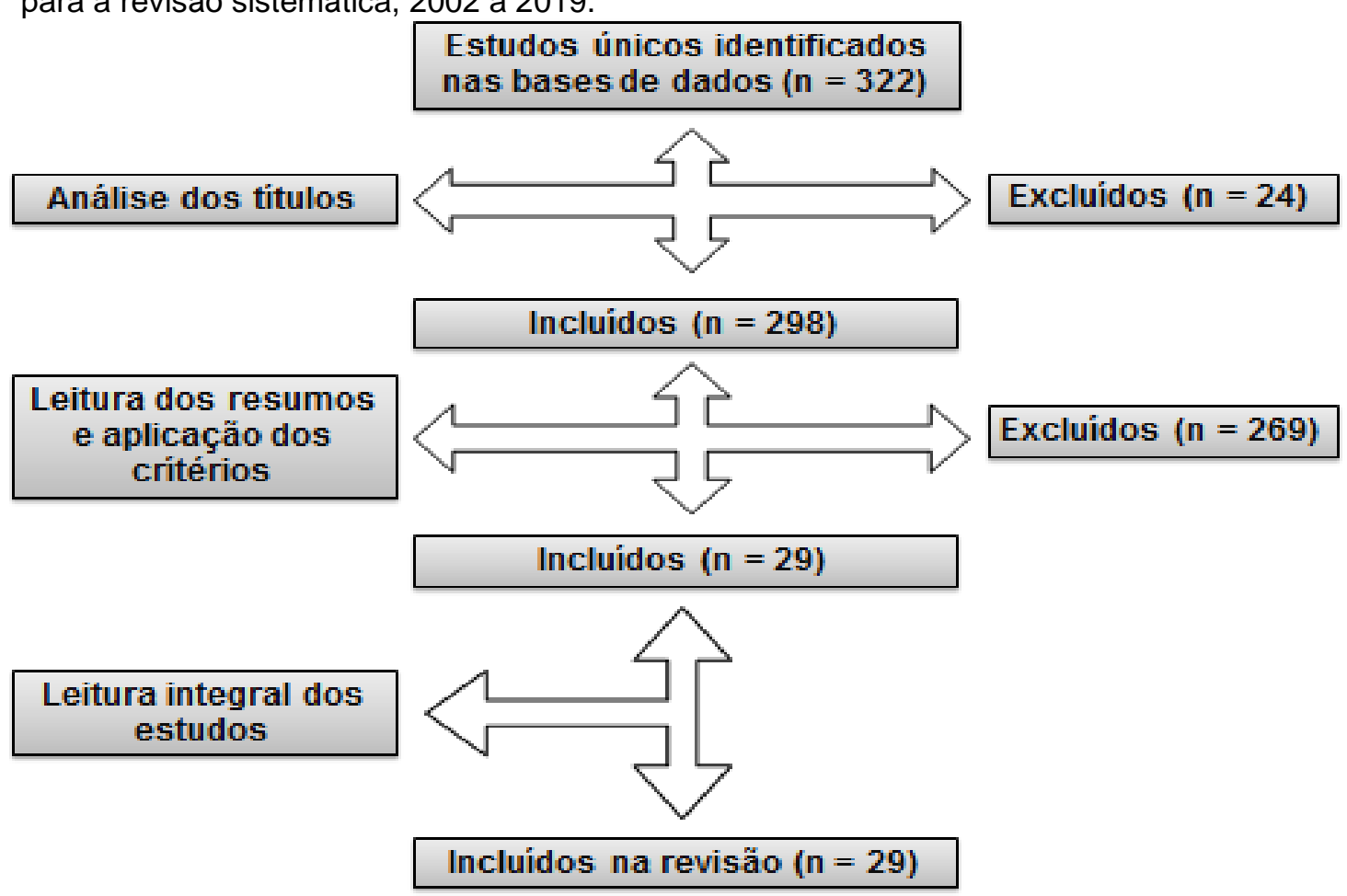

Fonte: Lessa RT, et al., 2020.

\section{RESULTADO E DISCUSSÃO}

A seguir são apresentadas informações gerais sobre os 29 estudos incluídos, publicados no período de 2002 a 2019. Dezoito manuscritos foram divulgados em periódicos classificados como Qualis Nacional "A2" ou superior. Os estudos utilizaram como instrumento de pesquisa questionários específicos para a coleta de dados, análise de prontuários e experimentos diversos. A composição das amostras quanto à faixa etária, incluiu período gestacional, infância, adolescência, vida adulta e velhice (Quadro 1). 


\section{Revista Eletrônica Acervo Saúde / Electronic Journal Collection Health ｜ ISSN 2178-2091}

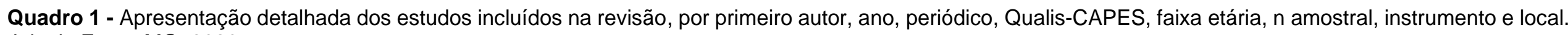
Juiz de Fora - MG, 2020.

\begin{tabular}{|c|c|c|c|c|c|c|c|}
\hline Primeiro autor & Ano & Periódico & $\begin{array}{l}\text { Qualis } \\
\text { CAPES }\end{array}$ & Faixa Etária & N Amostral & Instrumento & Local \\
\hline 1. JOMEEN J & 2007 & $\begin{array}{l}\text { Journal of Reproductive and } \\
\text { Infant Psychology }\end{array}$ & $\mathrm{A} 2$ & $\begin{array}{l}\text { Idade média } \\
28,86 \text { anos }\end{array}$ & 148 gestantes & $\begin{array}{l}\text { Questionários e análise de } \\
\text { prontuários }\end{array}$ & Hull, Reino Unido \\
\hline 2. YU Y & 2017 & $\begin{array}{c}\text { Archives of Women's } \\
\text { Mental Health }\end{array}$ & $\mathrm{A} 2$ & $\begin{array}{l}\text { Idade média } \\
27,90 \text { anos }\end{array}$ & 1653 gestantes & $\begin{array}{l}\text { Questionários e análise de } \\
\text { prontuários }\end{array}$ & Zhejiang, China \\
\hline $\begin{array}{l}\text { 3. TOMFOHR- } \\
\text { MADSEN L }\end{array}$ & 2019 & $\begin{array}{l}\text { Journal of Health } \\
\text { Psychology }\end{array}$ & B1 & $\begin{array}{l}\text { Idade média } \\
28,70 \text { anos }\end{array}$ & 290 gestantes & $\begin{array}{c}\text { Actigrafia, questionários e } \\
\text { análise de prontuários } \\
\text { eletrônicos }\end{array}$ & - \\
\hline 4. OKUN ML & 2013 & Psychosomatic Medicine & $\mathrm{A} 2$ & $\begin{array}{l}\text { Entre } 15 \text { e } 44 \\
\quad \text { anos }\end{array}$ & 168 gestantes & Questionários & $\begin{array}{c}\text { Cleveland e } \\
\text { Pittsburgh, Estados } \\
\text { Unidos }\end{array}$ \\
\hline 5. ZHONG C & 2018 & Sleep Medicine & $\mathrm{A} 1$ & $\begin{array}{c}\text { Idade média } 30 \\
\text { anos }\end{array}$ & 4066 gestantes & Questionários & Wuhan, China \\
\hline 6. BIAN SS & 2017 & $\begin{array}{l}\text { The Journal of Biological } \\
\text { Chemistry }\end{array}$ & A2 & $\begin{array}{l}72 \text { horas pós } \\
\text { fertilização }\end{array}$ & $\begin{array}{l}33 \text { Embriões } \\
\text { de peixe-zebra }\end{array}$ & $\begin{array}{c}\text { Experimentos com embriões } \\
\text { de peixe-zebra utilizando } \\
\text { medicamentos }\end{array}$ & Chengdu, China \\
\hline 7. KUDOVA J & 2016 & Journal of Pineal Research & $\mathrm{A} 1$ & - & - & $\begin{array}{l}\text { Introdução de células-tronco } \\
\text { embrionárias em ratos }\end{array}$ & Califórnia, EUA \\
\hline 8. NOGUEIRA RC & 2017 & $\begin{array}{c}\text { Journal of Experimental } \\
\text { Biology }\end{array}$ & B1 & $\begin{array}{c}\text { Dia } 0,5 \text { e } 15 \\
\text { pós fecundação }\end{array}$ & $\begin{array}{c}66 \text { embriões de } \\
\text { galinha }\end{array}$ & $\begin{array}{c}\text { Experimentos com embriões } \\
\text { de galinha utilizando } \\
\text { medicamentos }\end{array}$ & Belém, Brasil \\
\hline 9. JAMES S & 2017 & The Journal of Pediatrics & $\mathrm{A} 2$ & Até 9 anos & 1,567 crianças & $\begin{array}{c}\text { PCR e relatos de duração de } \\
\text { noites de sono. }\end{array}$ & Princeton, EUA \\
\hline 10. FALLONE G & 2005 & Sleep Research Society & $\mathrm{A} 2$ & $6-12$ anos & 74 crianças & Questionários & Providence, EUA \\
\hline 11. DUTIL C & 2017 & Journal of Sleep Research & $\mathrm{A} 2$ & Até 19 anos & 23 estudos & Literature Search & - \\
\hline 12. VON KRIES R & 2002 & $\begin{array}{c}\text { International Journal of } \\
\text { Obesity }\end{array}$ & $\mathrm{A} 1$ & $5-6$ anos & 6862 crianças & IMC, questionários & Bavária \\
\hline $\begin{array}{l}\text { 13. DE MORAES } \\
\text { MVP }\end{array}$ & 2011 & $\begin{array}{l}\text { Revista Paulista de } \\
\text { Pediatria }\end{array}$ & B4 & $\begin{array}{l}\text { Idade média } \\
\qquad 17,5\end{array}$ & 600 estudantes & $\begin{array}{c}\text { Regressão logística binária, } \\
\text { adotando-se a ocorrência do } \\
\text { sobrepeso e obesidade. }\end{array}$ & Caruaru, Brasil \\
\hline 14. WIDOME R & 2019 & Childhood Obesity & B1 & $\begin{array}{l}\text { Alunos do } 90 \\
\text { ano }\end{array}$ & $\begin{array}{c}2134 \\
\text { estudantes }\end{array}$ & $\begin{array}{c}\text { Avaliação da duração do } \\
\text { sono autorreferido nas noites } \\
\text { escolares associado ao peso } \\
\text { e sobrepeso }\end{array}$ & Minnesota, EUA \\
\hline
\end{tabular}

REAS/EJCH | Vol.Sup.n.56 | e3846 | DOI: https://doi.org/10.25248/reas.e3846.2020 Página 4 de 10 
Revista Eletrônica Acervo Saúde / Electronic Journal Collection Health ISSN 2178-2091

\begin{tabular}{|c|c|c|c|c|c|c|c|}
\hline Primeiro autor & Ano & Periódico & $\begin{array}{l}\text { Qualis } \\
\text { CAPES }\end{array}$ & Faixa Etária & N Amostral & Instrumento & Local \\
\hline 15. MATHIAS A & 2006 & UNESP & B4 & $\begin{array}{c}1^{\mathrm{a}}, 2^{\mathrm{a}} \text { e } 3^{\mathrm{a}} \\
\text { séries do } \\
\text { Ensino Médio } \\
\end{array}$ & 92 alunos & $\begin{array}{c}\text { Material didático - } \\
\text { pedagócico confeccionado } \\
\text { pelos próprios autores }\end{array}$ & Assis, Brasil \\
\hline 16. JUNIOR GJF & 2019 & $\begin{array}{c}\text { Revista Paulista de } \\
\text { Pediatria }\end{array}$ & B4 & 14 à 19 anos & 773 alunos & $\begin{array}{c}\text { Pediatric Daytime Sleepiness } \\
\text { Scale (PDSS) }\end{array}$ & Paranaguá, Brasil \\
\hline 17. BOSCOLO RA & 2007 & $\begin{array}{l}\text { Revista Portuguesa de } \\
\text { Ciências do Desporto }\end{array}$ & B4 & 12 à 14 anos & 45 alunos & Questionários & Santo André, Brasil \\
\hline 18. AYAS NT & 2003 & $\begin{array}{c}\text { Archiever of Internal } \\
\text { Medicine }\end{array}$ & - & $45-65$ anos & 71617 & Questionários & EUA \\
\hline 19. WALKER M & 2018 & - & - & - & - & Livro & - \\
\hline 20. IRWIN M & 2002 & $\begin{array}{l}\text { Brain, behavior, and } \\
\text { immunity }\end{array}$ & $\mathrm{A} 1$ & - & - & Literatura & - \\
\hline 21. GANGWISCH JE & 2014 & Sleep & $\mathrm{A} 1$ & $32-86$ anos & 8992 & $\begin{array}{c}\text { Análise do sono e } \\
\text { diagnóstico médico e } \\
\text { laboratorial }\end{array}$ & EUA \\
\hline 22. WU Y & 2014 & Sleep Medicine & $\mathrm{A} 2$ & Adult (over 18) & 361.922 & Meta-análise & - \\
\hline 23. JENSEN TK & 2013 & $\begin{array}{l}\text { American Journal of } \\
\text { Epidemiology }\end{array}$ & $\mathrm{A} 1$ & $\begin{array}{l}\text { Adult (over } 18 \\
\text { and male) }\end{array}$ & 953 & Exames variados & $\begin{array}{c}\text { University of } \\
\text { Southern Denmark }\end{array}$ \\
\hline 24. KLOSS JD & 2014 & Sleep medicine reviews & $\mathrm{A} 1$ & $\begin{array}{l}\text { Adult (over } 18 \\
\text { and female) }\end{array}$ & 7643 & Exames variados & EUA \\
\hline $\begin{array}{l}\text { 25. MÖLLER-LEVET } \\
\text { CS }\end{array}$ & 2013 & $\begin{array}{l}\text { Proceedings of the National } \\
\text { Academy of Sciences }\end{array}$ & $\mathrm{A} 1$ & (2) & - & - & Dallas, EUA \\
\hline 26. DE SÁ RMB & 2007 & $\begin{array}{c}\text { Revista Brasileira de } \\
\text { Geriatria e Gerontologia }\end{array}$ & B4 & $\begin{array}{l}60 \text { anos ou } \\
\text { mais }\end{array}$ & 105 idosos & Revisão de Prontuário & $\begin{array}{c}\text { Rio de Janeiro, } \\
\text { Brasil } \\
\end{array}$ \\
\hline 27. DIDIKOGLU A & 2019 & Journal of Sleep Research & $\mathrm{A} 2$ & $65-83$ & 6,375 & Questionário & $\begin{array}{c}\text { Greater Manchester } \\
\text { and Newcastle }\end{array}$ \\
\hline 28. FERRETTI F & 2018 & Brazilian Journal of Pain & - & 60 a 69 anos & 385 & $\begin{array}{c}\text { Mini-exame de estado } \\
\text { mental, instrumento } \\
\text { adaptado de Morais, escala } \\
\text { visual numérica e o } \\
\text { questionário de Pittsburgh. }\end{array}$ & $\begin{array}{c}\text { Santa Catarina, } \\
\text { Brasil }\end{array}$ \\
\hline 29. LUCEY BP & 2019 & $\begin{array}{c}\text { Science Translational } \\
\text { Medicine }\end{array}$ & $\mathrm{A} 1$ & $\begin{array}{l}\text { Maiores de } 60 \\
\text { anos }\end{array}$ & 119 & Clinical Dementia Rating & St. Louis, EUA \\
\hline
\end{tabular}

Fonte: Lessa RT, et al., 2020. 
O sono insuficiente em gestantes está correlacionado a um conjunto de fatores, principalmente depressão e ansiedade. Em um estudo realizado nos Hospitais Hull e East Yorkshire, na Inglaterra, analisou-se a saúde mental das gestantes com sono precário, com base no Índice de Qualidade do Sono de Pittsburgh (PSQI) e na Escala de Depressão Pós-Natal de Edinburgh, em que as gestantes, majoritariamente, apresentaram depressão no primeiro trimestre da gravidez (JOMEEN J e MARTIN CR, 2007).

Dados semelhantes foram identificados em uma pesquisa transversal e longitudinal do sono, com a depressão e ansiedade associadas, entre as mulheres grávidas chinesas no Zhoushan Maternal and Child Care Hospital no período entre 2011 e 2015, em que consistia na avaliação das gestantes em cada trimestre, sendo possível observar uma maior incidência de depressão e ansiedade no primeiro deles (YU Y, et al., 2017). Nesse contexto é importante salientar que a ansiedade na gestação interagiu com a duração do sono, de tal modo que a ansiedade na gravidez esteve significativamente associada ao parto prematuro, quando as mulheres tiveram uma duração relativamente curta do sono (aproximadamente $<8,3$ horas) (TOMFOHRMADSEN L, et al., 2019).

Um fato importante desencadeado pela depressão é o aumento das concentrações de citocinas inflamatórias circulantes na gestante e suas consequências. Além disso, inflamação crônica é um fator de risco para vários problemas adversos à saúde da grávida, desfechos incluindo doenças cardiovasculares e síndrome metabólica. Um estudo realizado pelo Instituto de Medicina do Sono e Departamento de Psiquiatria da Universidade de Pittsburgh, verificou que entre as mulheres deprimidas, a curta duração do sono $(<7$ horas) foi associada a maior concentração de interleucina (IL) -8 e (IL) -6 ao longo do tempo; e cochilos diurnos foram associados a um maior fator de necrose tumoral (TNF) $\alpha$. Para mais, aspectos relacionados a um sono insuficiente foram associados ao peso dos bebês, um aumento da IL-6, foi referente a um baixo peso dos recém- nascidos, no caso da IL-8, os bebês apresentaram maior peso (OKUN ML, et al., 2013).

Outra variável relacionada à má qualidade do sono no primeiro trimestre da gravidez é o aumento do desenvolvimento de Diabetes Mellitus Gestacional (DMG). Em um estudo com 4066 mulheres grávidas, dentre as quais um total de $335(8,2 \%)$ participantes foram diagnosticadas com DMG. Também, a má qualidade do sono foi relatada em 259 (6,4\%) mulheres no início da gestação e 248 (6,1\%), no meio dela. Desse modo, as variáveis que irão potencializar os efeitos adversos do sono precário no desenvolvimento de DMG serão: o avanço da idade materna, histórico familiar de diabetes e os próprios riscos da DMG (ZHONG C, et al., 2018).

Além de tudo, o progresso da pesquisa em melatonina, um mensageiro do ritmo circadiano entre mãe e feto, revelou que ela desempenhou um papel importante na diferenciação cardíaca e na maturação das células-tronco embrionárias; e na formação do coração em forma de $S$ do embrião. Dessa forma, conclui-se a relação do sono materno com um risco aumentado de doença coronariana, felizmente, a concordância com a soneca diurna pode, em certa medida, enfraquecer o risco na doença arterial coronariana simples (BIAN SS, et al., 2017; KUDOVA J, et al., 2016; NOGUEIRA RC e SAMPAIO LFS, 2017).

Estudos demonstraram que a privação do sono durante a infância denota em consequências nocivas na esfera microscópica de um indivíduo, tanto que, crianças com decréscimos no tempo noturno de sono tendem a possuir telômeros de configuração mais curta em detrimento daquelas que obtiveram um sono plenamente suficiente ( 0.015 log-kilobases menor por cromossomo em detrimento ao tamanho usual). James $\mathrm{S}$, et al. (2017); sustentam que telômeros mais curtos explicitariam um mecanismo ou indicariam um caminho biológico, revelando a correlação entre a duração do sono na infância e sua saúde e bem-estar a longo prazo.

No estudo de Fallone G, et al. (2005), nota-se uma restrição de oportunidades de sono em crianças saudáveis, em vida escolar normal. Durante um período de uma semana, aumentaram-se as avaliações de professores sobre dificuldades acadêmicas em sala, assim como problemas de atenção vinculados à escola. Professores indicaram que as crianças estavam mais lentas no processamento de novas informações e com propensões ao esquecimento, em condições de restrição do sono. Além disso, os educadores, ao notarem problemas acadêmicos, os designavam justamente ao grupo de indivíduos submetidos à privação de sono, fato esse que corrobora com a literatura de que a menor falta de sono acarreta na aparente dificuldade de aprendizado. 
Dutil C e Chaput JP (2017) fornecem contribuições notórias ao inferir uma inadequação do sono como fator predisponente de Diabetes Mellitus Tipo 2, em crianças. Através dos padrões de sono, obtém-se uma melhor compreensão da associação entre o sono e a homeostase da glicose em crianças e adolescentes. Da mesma forma, evidências sugerem que a curta duração do sono e sua má qualidade estão associadas à resistência à insulina, em crianças e adolescentes. Ainda acerca do sono inadequado na infância, Von Kries $\mathrm{R}$, et al. (2002) demonstraram que os dados de crianças na Bavária estabeleceram uma relação de dose-dependência na prevalência de sobrepeso e obesidade, com a duração do período de sono, com enfoque nas crianças de 5-6 anos. Apura-se que dormir diariamente em torno de 11,5 horas ou mais reduz o risco de obesidade e excesso de massa lipídica, nesta faixa etária, pela metade ou ainda menos.

O sono diminuto sugestiona alterações no padrão alimentar e de atividade física, culminando em um aumento de sobrepeso e obesidade no adolescente. Nesse sentido, em um estudo transversal realizado com jovens pernambucanos, foi possível identificar maior proporção de obesos entre aqueles com baixa qualidade do sono, os insuficientemente ativos, os que relataram assistir TV por mais de três horas por dia e com consumo de doce igual ou superior a quatro vezes por semana $(p<0,05)$ (DE MORAES MVP et al., 2011).

Diante dessa problemática social, outro estudo conduzido por Widome R, et al. (2019) analisou a duração do sono durante as noites escolares em associação com comportamentos relacionados ao peso (alimentação, dieta e atividade física), sobrepeso e obesidade em adolescentes. Constatou-se, que $85 \%$ dos jovens analisados relataram que não tinham uma duração de sono suficiente. Assim, a menor duração do sono esteve associada ao maior consumo de carboidratos, cafeína, energéticos e ingestão reduzida de legumes. Aqueles que relataram durações mais curtas do sono eram menos propensos a relatar atividade física em pelo menos 3 dias por semana. Os que possuíam horas dormidas abaixo de 7:00 à 7:30h eram mais propensos a ter um IMC na faixa de sobrepeso ou obesidade.

Ainda, uma das principais consequências da privação do sono em adolescentes é a sonolência diurna. Em um estudo realizado por Mathias A, et al. (2006) houve um levantamento sobre o padrão de sono em estudantes do Ensino Médio, dentre as queixas mais recorrentes, estava a sonolência diurna com 47,8\%.

Outro estudo, liderado por Junior GJF, et al. (2019) avaliou a sonolência diurna decorrente da necessidade subjetiva de sono em adolescentes e comparou aspectos do sono e índice de massa corporal entre os que consideram dormir o suficiente e os que julgam necessitar dormir mais. A partir disso, reuniram jovens de 14 a 19 anos e obteve-se uma prevalência de adolescentes com necessidade subjetiva de sono de $73 \%$, sugerindo precisarem, em média, de 1,7 horas a mais de sono, ratificando, portanto, o estudo anterior. Dessa forma, a necessidade subjetiva de sono foi a variável com maior poder explicativo da sonolência diurna $(24,8 \% ; p<0,001)$. Além disso, quanto menos os adolescentes praticavam atividade física, maiores as pontuações de sonolência diurna.

Vale salientar também que na pesquisa realizada por Boscolo RA, et al. (2007) foi verificada a relação da qualidade do sono com a função cognitiva dos adolescentes. Os dados sugerem que os alunos com menos queixas de sono demonstraram melhor desempenho nas recordações de palavras, enquanto que os estudantes submetidos ao turno matutino apresentaram uma redução na duração de sono. Esses achados demonstram que possivelmente o período de estudo e os hábitos de sono estão interligados e podem influenciar no desempenho escolar de adolescentes.

A privação do sono durante a vida adulta de maneira crônica está comprovadamente associada a diversos quadros de desequilíbrio fisiológico e a várias doenças. Exemplo disso é um estudo conduzido por Ayas NT, et al. (2003) que concluiu que pessoas que dormem 5 horas ou menos diariamente foram associadas a um aumento de $39 \%$ no risco de desenvolver doenças coronarianas em comparação com indivíduos que possuem sono de 8 horas diárias. Esses efeitos se devem, majoritariamente, ao funcionamento desregulado do Sistema Nervoso Autônomo Simpático por falta de suspensão da adrenalina, neurotransmissor desse sistema, o que deveria ocorrer todas as noites durante um sono satisfatório (WALKER M, 2018).

Outro estudo, dessa vez associando a falta de sono ao sistema imunitário adulto, comprovou o efeito imunossupressor da permanência prolongada em vigília. Somente uma quantidade modesta de privação de sono impactou a contagem de células imunitárias e alterou a secreção noturna de citocinas pró-inflamatórias, como interleucina-6 (IRWIN M, 2002). 
Contudo, o excesso de sono também pode se mostrar maléfico. Nessa situação, diversas substâncias reguladoras são descompensadas, inclusive diminuindo a demanda de glicose pelo cérebro e aumentando a intolerância à glicose ingerida. No estudo de Gangwisch JE, et al. (2007) indivíduos que dormiram 5 horas ou menos por dia obtiveram $47 \%$ mais chance de desenvolverem diabetes ao longo dos 10 anos seguintes, em comparação com um grupo controle. Além disso, aquelas pessoas que dormiram 9 horas ou mais obtiveram $52 \%$ mais chance de contraírem diabetes nas mesmas condições.

Com respeito à obesidade, o estudo de meta-análise conduzido por Wu $\mathrm{Y}$, et al. (2014) envolvendo 197.906 indivíduos, revelou que a curta duração de sono está fortemente relacionada à futura apresentação de obesidade. Todavia, o contrário, ou seja, a longa duração de sono, não está relacionada da mesma forma.

Como era de se esperar, a falta de sono também pode prejudicar a saúde sexual. Os efeitos da privação de sono levaram a uma redução de $25 \%$ na contagem total de espermatozoides em homens com graves distúrbios e consequente privação crônica do sono, numa amostragem com 953 jovens dinamarqueses saudáveis (JENSEN TK, 2013). Já nas mulheres, o sono deficiente pode causar infertilidade e problemas na concepção, devido ao descompasso de hormônios reprodutivos femininos (KLOSS JD, 2014). Logo, o estudo conduzido por Möller-Levet CS, et al. (2013) demonstrou que a qualidade de sono do indivíduo altera a expressão de suas estruturas gênicas. A partir de amostras de transcriptoma do sangue, os pesquisadores concluíram que 711 genes foram hipo ou hiper estimulados pelo sono insuficiente numa amostragem de 26 pessoas, dentre esses, genes relacionados ao ritmo circadiano, homeostase do sono, estresse oxidativo e metabolismo em geral.

Diversos estudos associam a privação do sono às doenças cardiovasculares, quedas noturnas, incidência de Alzheimer e problemas relacionados à saúde mental em idosos. Vislumbra-se que, além desses, a dor crônica, que não tem sua gênese na privação do sono, exerce influência na sua efetividade. Quadros de dor são associados à redução da motivação, apatia social e diminuição do bem-estar, fatores que se acumulam à comorbidades inerentes do avançar da idade e prejudicam ainda mais o padrão do sono (DE SÁ RMB, et al., 2007; DIDIKOGLU A, et al., 2019).

Estudo conduzido por Ferretti $F$, et al. (2019) baseado na relação entre qualidade do sono e incidência de dor crônica em população idosa demonstrou que, dentre as doenças de afecções nos idosos, a dor configurase como uma das maiores queixas relatadas por $58,18 \%$ deles. Além disso, os autores abordam que a dor crônica torna-se mais um elemento significativo na redução da qualidade do sono, e consequentemente, acarreta prejuízos a seu bem-estar. Dentre os idosos que se queixaram de dor crônica, $57,59 \%$ apresentaram qualidade de sono ruim, e aqueles que não possuíam dor crônica apresentaram qualidade de sono satisfatória, com $\mathrm{p}=0,000$. Portanto, o padrão de sono é alterado pela recorrência de dores crônicas, gerando um ciclo vicioso, no qual a sensação somestésica referida piora-o, e a queda do sono decai o ritmo do idoso, findando-o por sentir mais dor.

De Sá RMB, et al. (2007) evidenciaram, através da análise de dados apurada, que $85 \%$ dos pacientes com insônia apresentaram queixa de cansaço diurno intenso e 73,53\% demonstraram que a dificuldade para dormir deprecia muito em suas atividades da vida diária, no humor, memória, concentração e em labutas. Sabe-se que o desvio conhecido como insônia não é apenas um decréscimo na quantidade de horas de sono, mas também uma progressiva queda na qualidade de horas dormidas. Ainda tomando como base o estudo supracitado, nota-se que fatores de risco como depressão, ansiedade, incontinência urinária e prostatismo, osteoartrose, Doença Vascular Periférica (DVP), demência, osteoporose, Doença Pulmonar Obstrutiva Crônica (DPOC), dor crônica e Doença do Refluxo Gastroesofágico (DRGE) estão intimamente atrelados à queixa de insônia com significância estatística $(p<0,05)$.

Corroborando com o estudo previamente analisado, Didikoglu A, et al. (2019) concluíram que, em pacientes idosos com menor eficiência do sono, problemas respiratórios (DPOC) são recorrentes, além de inferir que distúrbios circulatórios são $14 \%$ menos frequentes em pacientes que possuem uma recomendada qualidade do sono $(p<0,001)$. Ademais, as queixas de hipertensão foram $12 \%$ menores em idosos que possuem um número de fragmentações do sono reduzidas. A privação ou a escassez de horas de sono é também abordada em âmbito da saúde mental, posto que o risco de incidência de depressão foi $24 \%$ maior em pacientes com baixa eficiência longitudinal de sono. 
Lucey BP, et al. (2019) asseguram a inversa relação entre idade/qualidade de sono (visível na fase específica, NREM) e o aparecimento precoce de Alzheimer com subsequente morte neuronal, perdas sinápticas e atrofia cerebral. Foi comprovado que há um declínio do NREM com a progressão da idade e sugere-se que a qualidade de sono piora com o aumento da deposição de complexo protéico TAU, gerando um efeito de retroalimentação positiva entre eficiência de sono e precocidade do Alzheimer.

\section{CONSIDERAÇÕES FINAIS}

O sono demonstrou ser um grande regulador da homeostase humana, em razão da sua imensurável pertinência no revigoramento dos sistemas do corpo. À vista disso, a privação do sono está comprovadamente associada ao desenvolvimento sistêmico de diversas doenças em todas as fases da vida. Sendo importante salientar o desenvolvimento de Diabetes Mellitus, em adultos, crianças e de forma mais relevante, no grupo de mulheres grávidas. Outro preditivo seria o maior risco de sobrepeso e obesidade em adolescentes, assim como a resistência à insulina e déficits na aprendizagem observados nesse grupo e também em infantos. Somando-se a isso, em crianças, foi relatada a ocorrência de telômeros com configuração mais curta, devido ao decréscimo no tempo de sono. Em gestantes, a associação entre a queda de sono como desencadeadora da ansiedade foi importante para elucidar a incidência de partos prematuros. Já em adultos, foi destaque a manifestação de doenças coronarianas e imunossupressão, sendo pertinente ser dito que em idosos, a dor crônica é acentuada pela privação de sono, enquanto que essa também influencia nos episódios de dor, caracterizando um infeliz ciclo vicioso.

\section{AGRADECIMENTOS E FINANCIAMENTO}

Os autores agradecem pelo apoio financeiro à publicação desse artigo ao Diretório Acadêmico Dr. Olamir Rossini - DAOR, do Centro Universitário Presidente Antônio Carlos - UNIPAC-JF.

\section{REFERÊNCIAS}

1. AYAS NT, et al. A prospective study of sleep duration and coronary heart disease in women. Arch intern med. 2003; 163(2): 205-209.

2. BIAN SS, et al. Clock1a affects mesoderm development and primitive hematopoiesis by regulating nodal-Smad3 signaling in the zebrafish embryo. The Journal of Biological Chemistry. 2017; 292(34): 14165-14175.

3. BOSCOLO RA, et al. Avaliação do padrão de sono, atividade física e funções cognitivas em adolescentes escolares. Revista portuguesa de ciências do desporto. 2007; 7(1): 18-25.

4. BRYANT PA, et al. Sick and tired: does sleep have a vital role in the immune system? Nature Reviews Immunology. 2004; 4(6): 457.

5. DE MORAES MVP, et al. Fatores associados ao sobrepeso e à obesidade em estudantes do ensino médio da rede pública estadual do município de Caruaru (PE). Revista paulista de pediatria. 2011; 29 (4): 536-545.

6. DE SÁ RMB, et al. Insônia: prevalência e fatores de risco relacionados em população de idosos acompanhados em ambulatório. Rev. Bras. Geriatr. Gerontol. 2007; 10(2): 217-230

7. DIDIKOGLU A, et al. Longitudinal sleep efficiency in the elderly and its association with health. J Sleep Res. 2019; 00: e12898.

8. DUTIL C, CHAPUT JP. Inadequate sleep as a contributor to type 2 diabetes in children and adolescents. Nutrition \& Diabetes. 2017; 7(5): 266.

9. FALLONE G, et al. Experimental restriction of sleep opportunity in children: effects on teacher ratings. Sleep. 2005; 28:1561-1567;

10. FERRETTI F, et al. Sleep quality in the elderly with and without chronic pain. Br J Pain. 2018; 1(2): 141-146.

11. GANGWISCH JE, et al. Sleep duration as a risk factor for diabetes incidence in a large US sample. Sleep. 2007; 30(12): 1667-1673.

12. IRWIN M. Effects of sleep and sleep loss on immunity and cytokines. Brain, behavior, and immunity. 2002; 16(5): 503512.

13. JAMES S, et al. Sleep Duration and Telomere Length in Children. The Journal of Pediatrics. 2017; 187: $247-252$.

14. JANSEN JM, et al. Medicina da noite: da cronobiologia à prática clínica. SciELO - Fiocruz: Rio de Janeiro. 2007.

15. JENSEN TK, et al. Association of sleep disturbances with reduced semen quality: a cross sectional study among 953 healthy young Danish men. Am J Epidemiol. 2013; 177(10): 1027-1037.

16. JOMEEN J, MARTIN CR. Assessment and relationship of sleep quality to depression in early pregnancy. Journal of Reproductive and Infant Psychology. 2007; 25(1): 87-99.

17. JUNIOR GJF, et al. Necessidade subjetiva de sono e sonolência diurna em adolescentes. Revista Paulista de Pediatria. 2019; 37 (2): 209-216. 
18. KLOSS JD, et al. Sleep, sleep disturbance, and fertility in women. Sleep medicine reviews. 2014; 22(1): 78-87.

19. KUDOVA J, et al. Melatonin promotes cardiomyogenesis of embryonic stem cells via inhibition of HIF-1alpha stabilization. Journal of Pineal Research. 2016; 61(4): 493-503.

20. LUCEY BP, et al. Reduced non-rapid eye movement sleep is associated with tau pathology in early Alzheimer's disease. Sci. Transl. Med. 2019; 11(474), eaau6550.

21. MATHIAS A, et al. Incentivar hábitos de sono adequados: um desafio para os educadores. Núcleos de Ensino da Unesp. São Paulo: UNESP. 2006: 718-731.

22. MÖLLER-LEVET CS, et al. Effects of insufficient sleep on circadian rhythmicity and expression amplitude of the human blood transcriptome. Proceedings of the National Academy of Sciences. 2013; 110(12): 1132-1141.

23. NOGUEIRA RC, SAMPAIO LFS. Eye and heart morphogenesis are dependent on melatonin signaling in chick embryos. The Journal of Experimental Biology. 2017; 220(20): 3826-3835.

24. OKUN ML, et al. Disturbed Sleep and Inflammatory Cytokines in Depressed and Nondepressed Pregnant Women: An Exploratory Analysis of Pregnancy Outcomes. Rev. Psychosomatic Medicine. 2013; 75(7): 670-681.

25. TOMFOHR-MADSEN L, et.al. Pregnancy anxiety and preterm birth: The moderating role of sleep. Rev. Health Psychol. 2019; 38(8): 94-105.

26. TONONI G, CIRELLI C. Sleep function and synaptic homeostasis. Sleep Medicine Reviews. 2006; 10(1): 49-62.

27. VON KRIES R, et al. Reduced risk for overweight and obesity in 5- and 6-y-old children by duration of sleep-a crosssectional study. International Journal of Obesity. 2002; 26(5): 710-716.

28. WALKER M. Definição e geração de sono: Dilatação do tempo e o que aprendemos com um bebê em 1952. In: Walker M. Por que nós dormimos: A nova ciência do sono e do sonho. 1 ed. Rio de Janeiro: Intrínseca, 2018; 51-69.

29. WALKER M. Dormir... In: Walker M. Por que nós dormimos: A nova ciência do sono e do sonho. 1 ed. Rio de Janeiro: Intrínseca, 2018; 15-24.

30. WIDOME R, et al. Sleep Duration and Weight-Related Behaviors among Adolescents. Childhood Obesity. $2019 ; 15$ (7): 434-442.

31. WU Y, et al. Sleep duration and obesity among adults: a meta-analysis of prospective studies. Sleep Medicine. 2014; 15(12): 1456-1462.

32. $Y U$ Y, et. al. Sleep was associated with depression and anxiety status during pregnancy: a prospective longitudinal study. Rev. Archives of Women's Mental Health. 2017; 20(5): 695-701.

33. ZHONG C, et al. Poor sleep during early pregnancy increases subsequent risk of gestational diabetes mellitus. Rev. Sleep Medicine. 2018; 46: 20-25. 